\title{
KRAS Mutations and Rectal Cancer Response to Chemoradiation: Are We Closer to Personalization of Therapy?
}

\author{
Sunil Krishnan, $\mathrm{MD}^{1}$ and George J. Chang, MD, $\mathrm{MS}^{2}$ \\ ${ }^{1}$ Department of Radiation Oncology, The University of Texas MD Anderson Cancer Center, Houston, TX; ${ }^{2}$ Department of \\ Surgical Oncology, The University of Texas MD Anderson Cancer Center, Houston, TX
}

Ras is a membrane-bound protein that regulates multiple downstream pathways, including the Raf/MAPK and the PI3K/Akt signaling pathways. ${ }^{1}$ When bound to guanosine triphosphate (GTP), it remains in an active state but is inactivated by intrinsic GTPase activity that hydrolyzes GTP to guanosine diphosphate (GDP). Ras is activated by upstream receptor tyrosine kinases, including epidermal growth factor receptor (EGFR), that recruit guanine nucleotide exchange factors (GEFs) to the cell membrane to open up the GTP binding site of Ras. Ras-GTPase then deactivates Ras with the assistance of GTPase activating proteins (GAPs). This intricate balance between activation and deactivation of Ras efficiently regulates the communication of signals arising from receptor tyrosine kinases on the cell membrane to effector molecules in the cytoplasm and nucleus. Mutations in the KRAS gene disrupt this fine balance.

Acquired mutations in KRAS are an early step in carcinogenesis, identified in approximately $40 \%$ of colorectal cancers. The most common mutations occur at codons 12 and 13 of exon 2 of the KRAS gene, which encode glycine. These mutations in the phosphate-binding loop of Ras deactivate its intrinsic GTPase activity and render it resistant to GAP-mediated GTP hydrolysis, thereby locking Ras into the activated state. Constitutively active Ras is no longer dependent on upstream activation of receptor tyrosine kinases and as a corollary, insensitive to antiEGFR chemotherapy. An abundance of literature confirming that the presence of KRAS mutation predicts lack of

(C) Society of Surgical Oncology 2013

First Received: 30 May 2013;

Published Online: 18 July 2013

G. J. Chang, MD, MS

e-mail: gchang@mdanderson.org response of colon cancers to anti-EGFR therapy has resulted in the increasing use of KRAS mutation analysis in clinical practice. However, its role as a prognostic marker of overall survival independent of anti-EGFR therapy remains less clear. ${ }^{2}$ This is especially true for rectal cancer where studies have shown conflicting results on the predictive value of KRAS mutation status on response to neoadjuvant chemoradiation therapy. This was corroborated by a meta-analysis showing no difference in pathologic complete response rates, tumor downstaging, or cancer-specific survival between KRAS wild-type and $K R A S$ mutant rectal cancer patients treated with chemoradiation irrespective of the use of anti-EGFR therapy. ${ }^{3}$ The authors of the accompanying manuscript have previously reported on the associations between KRAS mutations and pathologic complete response following neoadjuvant chemoradiation therapy for rectal cancer among patients treated on a parent study examining the effect of time interval to surgery following neoadjuvant chemoradiation therapy. ${ }^{4}$ In the present study, the authors explore this issue further by asking (1) whether the type of KRAS mutation has an impact on predicting treatment outcomes and (2) whether there are any notable associations between $K R A S$ and TP53 mutations that might explain the heterogeneity of treatment responses observed. ${ }^{5}$ We consider these two questions separately below.

Not all KRAS mutations are created equal. As noted by the authors in the accompanying manuscript, even though the native amino acid at both these codon loci (12 and 13) is glycine, missense mutations result in amino acids as varied as aspartate, valine, alanine, cysteine, and serine. The most common of these is the glycine $\rightarrow$ aspartate transition at both codons. The next most common missense mutation is the glycine $\rightarrow$ valine transversion (conversion of a purine to a pyrimidine). Because this transversion requires replacement of both the base and the amino acid, it 
may be the result of a greater genotoxic stress, which was suggested by a study of KRAS mutations in lung cancer patients where those who never smoked were more likely to have transitions (rather than transversions) than those who were former or current smokers. ${ }^{6}$ An analysis of 94 rectal cancer patients treated with preoperative chemoradiation therapy concluded that G12V mutations appeared to be associated with a lower rate of tumor regression than G13D mutations. ${ }^{7}$ Similar results were noted in Dukes B and $\mathrm{C}$ colon cancer patients where those harboring G12V mutations had shorter disease-free and overall survival. ${ }^{8}$ However, the emerging data on KRAS G13D mutation status and resistance to anti-EGFR therapy are inconsistent and conflicting with some studies, suggesting that the mutation confers patients with improved tumor response and progression-free survival with ant-EGFR therapy, whereas other do not. ${ }^{9-11}$

From a biologic standpoint, multiple lines of evidence suggest that tumor behavior and response to therapy of KRAS mutant cells are driven by the relative dominance of individual effector pathways downstream of Ras. First, fibroblasts transfected with mutant KRAS codon 12 are more resistant to apoptosis, more predisposed to anchorage-independent growth, and grow more readily as spheroids than fibroblasts transfected with mutant KRAS codon 13 or overexpressed wild-type Kras. ${ }^{12}$ This is partly explained by the higher association of codon 12 mutants with greater AKT activation compared with codon 13 mutants, which activated the JNK1 pathway more robustly. ${ }^{12}$ Second, although the influence of individual KRAS mutations on radiation resistance is inadequately studied, it is well known that Ras activation induces radiation resistance. ${ }^{13}$ Among individual downstream pathways of Ras, the dominant mediator of radiation resistance is the PI3K pathway and not the Raf-MEKMAPK or the Ras-MEK kinase-p38 pathway. ${ }^{14}$ Third, different mutant forms of KRAS codon 12 confer varying levels of chemosensitivity. In stably transfected non-smallcell lung cancer cell lines, the G12C mutant was more resistant to cisplatin and more sensitive to Taxol and pemetrexed compared with wild-type. In contrast, the G12V mutant was strongly sensitive to cisplatin and the G12D mutant was more resistant to Taxol. ${ }^{15}$ Taken together, these observations suggest that merely identifying mutated KRAS in a tumor may not provide sufficient information about the biologic activity or treatment responsiveness of a tumor; the individual mutation type plays an equally important role in determining treatment susceptibility, which may be mediated by the relative dominance of individual downstream effector pathways. One way to discern this would be assess the level of activation of each of these pathways in tumor tissues concurrently with mutation analyses. Alternatively, one may classify each mutation as one that disrupts a dominant pathway or one that does not. When TP53 mutations were analyzed in this fashion, patients with head and neck cancer who harbored disruptive TP53 mutations had a significantly higher risk of locoregional recurrence than those harboring no mutations or nondisruptive mutations. ${ }^{16}$ Dichotomizing TP53 mutation status as wild-type or mutant alone was not predictive of locoregional recurrence. In agreement with these biological rationales, the authors of the accompanying manuscript rightly conclude that $K R A S$ mutation status is not a binary variable and the specific mutation within the KRAS gene influences the heterogeneity of treatment responses observed.

The authors also postulate that specific mutations in KRAS do not occur in isolation and that the global genetic context modulates the impact of these mutations on overall treatment response of tumors. They identify a strong association between KRAS mutations in codon 13 and a high incidence $(75 \%)$ of a TP53 mutation. In contrast, only $37 \%$ of patients with any other KRAS mutation harbored a TP53 mutation. ${ }^{5}$ A similar interplay between $K R A S$ mutation and TP53 mutation was noted by Bazan et al. ${ }^{17}$ where the presence of TP53 mutations in the L3 domain in combination with KRAS mutations at codon 13 were predictive of worse prognosis in colorectal cancers. We surmise that, in the absence of pathway addiction by a driver mutation and specific targeting of the addicted pathway with a selective inhibitor, individual mutations in genes (passengers) may exert effects on treatment that are a consequence of their inherent ability to drive proliferative prosurvival pathways or occur in a context of other genetic defects that they either interact with or are overshadowed by to elicit a response to treatment. Thus, the variability in the resistance of KRAS mutant tumors to chemoradiation therapy may be due to inherent differences in downstream signaling by these KRAS mutant tumors and/or differences in prevalence of other mutations in these KRAS mutant tumors. Admittedly, these findings provide additional reasons to continue our quest to unearth genomic signatures that predict response of rectal cancers to preoperative chemoradiation therapy.

Many investigators have tried to identify molecular genetic predictors of $\mathrm{pCR}$ following CXRT. ${ }^{18-20}$ No definitive predictors exist, but as the authors have shown, the results have been more useful for predicting lack of response rather than to predict response. Other predictive modalities, such as imaging (MRI, PET) also have been studied but by themselves are limited. ${ }^{21-23}$ Nonetheless, it is important to continue to seek predictors of response to chemoradiation therapy, because response is a biomarker of excellent prognosis following TME and an excellent response may offer a real possibility of organ preservation, highlighting the importance of making the right 
decision. ${ }^{24-26}$ Unfortunately, current strategies, including evaluating clinical response and residual tumor within the bowel wall by local excision, are inadequate, particularly given the high stakes involved and the curative potential of TME. ${ }^{27}$

So how do we move forward? We envision a future where clinical, molecular genetic, and radiographic predictors are integrated into a composite decision-making algorithm with each additional factor increasing the pretest probability of the subsequently applied factor. Perhaps only then we can have sufficient predictive ability to confidently recommend organ-preserving treatment options for patients predicted to have a high probability of response and favorable prognosis without radical resection and treatment intensification (either chemo/biologic therapy, radiation therapy, or both) for patients predicted to have a low probability of response to chemoradiation therapy. In fact, identification of a signature of lack of response or resistance to therapy may be as useful as identification of a signature of treatment responsiveness. Furthermore, we expect that some of these biomarkers may not be selfevident until treatment has begun and a dynamic change from baseline triggered by the selection pressure of sublethal doses of chemoradiation therapy serves as a guide for decision-making and adaptive treatment modification. In a treatment-naïve situation, the signature of treatment resistant subpopulations may be drowned by that of the treatment sensitive subpopulations. Dynamic (inducible as opposed to constitutive) changes noted after initiating treatment might unmask the disproportionate influence of treatment-resistant subpopulations in dictating treatment failure. Similarly, accurate assessment of early changes in physiological imaging parameters that predate conventional morphological imaging parameters of responding (shrinking) tumors may not only predict subsequent clinicopathological measures of response but also offer the opportunity to make adaptive treatment modifications.

Thus, while the molecular mechanisms behind the authors' observations may not be clearly identified, the authors are to be commended for their commitment to advancing our understanding of the complex relationship between baseline molecular genetic characteristics of tumors and their response to neoadjuvant chemoradiation therapy. In this case KRAS mutations, either as driver or passenger, were informative regarding chemoradiation treatment response. Only with a rigorous approach to understanding the mechanisms of treatment response and resistance can we continue to make steps towards true treatment personalization.

DISCLOSURE Dr. Chang is supported in part by a National Cancer Institute Research Grant (K07-CA133187).

\section{REFERENCES}

1. Bardelli A, Siena S. Molecular mechanisms of resistance to cetuximab and panitumumab in colorectal cancer. J Clin Oncol. 2010;28(7):1254-61.

2. Allegra CJ, Jessup JM, Somerfield MR, et al. American Society of Clinical Oncology provisional clinical opinion: testing for $K R A S$ gene mutations in patients with metastatic colorectal carcinoma to predict response to anti-epidermal growth factor receptor monoclonal antibody therapy. J Clin Oncol. 2009; 27(12):2091-6.

3. Clancy C, Burke JP, Coffey JC. KRAS mutation does not predict the efficacy of neo-adjuvant chemoradiotherapy in rectal cancer: a systematic review and meta-analysis. Surg Oncol. 2013;22(2): $105-11$.

4. Garcia-Aguilar J, Chen Z, Smith DD, et al. Identification of a biomarker profile associated with resistance to neoadjuvant chemoradiation therapy in rectal cancer. Ann Surg. 2011;254(3):48692; discussion 492-3.

5. Duldulao MP, Lee W, Nelson RA, et al. Mutations in specific codons of the KRAS oncogene are associated with variable resistance to neoadjuvant chemoradiation therapy in patients with rectal adenocarcinoma. Ann Surg Oncol. 2013;20(7):2166-71.

6. Riely GJ, Kris MG, Rosenbaum D, et al. Frequency and distinctive spectrum of KRAS mutations in never smokers with lung adenocarcinoma. Clin Cancer Res. 2008;14(18):5731-4.

7. Gaedcke J, Grade M, Jung K, et al. KRAS and BRAF mutations in patients with rectal cancer treated with preoperative chemoradiotherapy. Radiother Oncol. 2010;94(1):76-81.

8. Andreyev HJ, Norman AR, Cunningham D, et al. Kirsten ras mutations in patients with colorectal cancer: the 'RASCAL II' study. Br J Cancer. 2001;85(5):692-6.

9. Tejpar S, Celik I, Schlichting M, Sartorius U, Bokemeyer C, Van Cutsem E. Association of KRAS G13D tumor mutations with outcome in patients with metastatic colorectal cancer treated with first-line chemotherapy with or without cetuximab. J Clin Oncol. 2012;30(29):3570-7.

10. De Roock W, Jonker DJ, Di Nicolantonio F, et al. Association of KRAS p.G13D mutation with outcome in patients with chemotherapy-refractory metastatic colorectal cancer treated with cetuximab. JAMA. 2010;304(16):1812-20.

11. Peeters M, Douillard JY, Van Cutsem E, et al. Mutant KRAS codon 12 and 13 alleles in patients with metastatic colorectal cancer: assessment as prognostic and predictive biomarkers of response to panitumumab. J Clin Oncol. 2013;31(6):759-65.

12. Guerrero S, Casanova I, Farre L, Mazo A, Capella G, Mangues R. K-ras codon 12 mutation induces higher level of resistance to apoptosis and predisposition to anchorage-independent growth than codon 13 mutation or proto-oncogene overexpression. Cancer Res. 2000;60(23):6750-6.

13. McKenna WG, Muschel RJ, Gupta AK, Hahn SM, Bernhard EJ. The RAS signal transduction pathway and its role in radiation sensitivity. Oncogene. 2003;22(37):5866-75.

14. Gupta AK, Bakanauskas VJ, Cerniglia GJ, et al. The Ras radiation resistance pathway. Cancer Res. 2001;61(10):4278-82.

15. Garassino MC, Marabese M, Rusconi P, et al. Different types of KRas mutations could affect drug sensitivity and tumour behaviour in non-small-cell lung cancer. Ann Oncol. 2011;22(1):235-7.

16. Skinner HD, Sandulache VC, Ow TJ, et al. TP53 disruptive mutations lead to head and neck cancer treatment failure through inhibition of radiation-induced senescence. Clin Cancer Res. 2012;18(1):290-300.

17. Bazan V, Migliavacca M, Zanna I, et al. Specific codon 13 K-ras mutations are predictive of clinical outcome in colorectal cancer 
patients, whereas codon $12 \mathrm{~K}$-ras mutations are associated with mucinous histotype. Ann Oncol. 2002;13(9):1438-46.

18. Ghadimi BM, Grade M, Difilippantonio MJ, et al. Effectiveness of gene expression profiling for response prediction of rectal adenocarcinomas to preoperative chemoradiotherapy. $J$ Clin Oncol. 2005;23(9):1826-38.

19. Pucciarelli S, Rampazzo E, Briarava M, et al. Telomere-specific reverse transcriptase (hTERT) and cell-free RNA in plasma as predictors of pathologic tumor response in rectal cancer patients receiving neoadjuvant chemoradiotherapy. Ann Surg Oncol. 2012;19(9):3089-96.

20. Watanabe T, Komuro Y, Kiyomatsu T, et al. Prediction of sensitivity of rectal cancer cells in response to preoperative radiotherapy by DNA microarray analysis of gene expression profiles. Cancer Res. 2006;66(7):3370-4.

21. Chang GJ, You YN, Park IJ, et al. Pretreatment high-resolution rectal MRI and treatment response to neoadjuvant chemoradiation. Dis Colon Rectum. 2012;55(4):371-7.

22. Kang JH, Kim YC, Kim H, et al. Tumor volume changes assessed by three-dimensional magnetic resonance volumetry in rectal cancer patients after preoperative chemoradiation: the impact of the volume reduction ratio on the prediction of pathologic complete response. Int J Radiat Oncol Biol Phys. 2010;76(4): $1018-25$.

23. Ruby JA, Leibold T, Akhurst TJ, et al. FDG-PET assessment of rectal cancer response to neoadjuvant chemoradiotherapy is not associated with long-term prognosis: a prospective evaluation. Dis Colon Rectum. 2012;55(4):378-86.

24. Park IJ, You YN, Agarwal A, et al. Neoadjuvant treatment response as an early response indicator for patients with rectal cancer. J Clin Oncol. 2012;30(15):1770-6.

25. Maas M, Nelemans PJ, Valentini V, et al. Long-term outcome in patients with a pathological complete response after chemoradiation for rectal cancer: a pooled analysis of individual patient data. Lancet Oncol. 2010;11(9):835-44.

26. Kosinski L, Habr-Gama A, Ludwig K, Perez R. Shifting concepts in rectal cancer management: a review of contemporary primary rectal cancer treatment strategies. CA Cancer J Clin. 2012; 62(3): 173-202.

27. Park IJ, You YN, Skibber JM, et al. Comparative analysis of lymph node metastases in patients with ypT0-2 rectal cancers after neoadjuvant chemoradiotherapy. Dis Colon Rectum. 2013; 56(2):135-41. 